\title{
Efficacy of the smaller target volume for stage III non-small cell lung cancer treated with intensity-modulated radiotherapy
}

\author{
XIANGCUN LIANG ${ }^{1,2}$, HUIMING YU ${ }^{2}$, RONG YU ${ }^{2}$, GANG XU² and GUANGYING ZHU ${ }^{2}$ \\ ${ }^{1}$ Department of Radiation Oncology, Hebei Provincial Chest Hospital, Shijiazhuang, Hebei 050041; \\ ${ }^{2}$ Department of Radiation Oncology, Peking University Cancer Hospital, Beijing 100142, P.R. China
}

Received February 11, 2015; Accepted June 17, 2015

DOI: $10.3892 / \mathrm{mco} .2015 .588$

\begin{abstract}
The present study reports the local recurrence, distant metastasis, progression-free survival, overall survival and radiation toxicity between two arms of stage III non-small cell lung cancer (NSCLC) treated with intensity-modulated radiotherapy (IMRT); one arm with clinical target volume (CTV) and the other without CTV. The two arms of local recurrence, distant metastasis, progression-free survival, overall survival, grade 3-4 radiation esophagitis and hematological toxicity had no statistical significance. However, the grade 3-4 radiation pneumonia rate of the group without CTV was significantly decreased. This supports the concept that stage III NSCLC treated with IMRT, which omitted CTV, can reduce the occurrence of radiation pneumonia. The aim of the present study was to analyze the feasibility of the smaller target volume for stage III NSCLC treated with IMRT. Data from 105 patients with stage III NSCLC who were hospitalized and received IMRT between January 1, 2008 and November 30, 2012 were retrospectively analyzed. A total of 55 cases were irradiated with target volume without CTV and 50 cases were irradiated with CTV. Dose prescription was $100 \%$ PTV at 54-63 Gy/27-35 F/5.4-7 weeks. The two arms of the patient characteristics and treatment deliveries had no statistical significance. The two arms of the patients were compared for local recurrence, distant metastasis, progression-free survival, overall survival and radiation-related toxicity. In the arms without and with CTV, the local relapse and distant metastases rates were 32.7 and $32.0 \%(\mathrm{P}=1.000)$ and 56.4 and $48.0 \%$ $(\mathrm{P}=0.946)$, respectively. The median progression-free survival time for the two arms was 9 months $(\mathrm{P}=0.619)$. The 1-, 2- and 3 -year survival rates of the arms without and with CTV were $74.5,43.6$ and $23.6 \%$, and 70.0, 46.0 and 20.0\% ( $\mathrm{P}=0.956)$, respectively. In the two arms, grade $3-4$ radiation esophagitis
\end{abstract}

Correspondence to: Professor Guangying Zhu, Department of Radiation Oncology, Peking University Cancer Hospital, 52 Fucheng Road, Beijing 100142, P.R. China

E-mail: zgypu@aliyun.com

Key words: stage III non-small cell lung cancer, intensity-modulated radiotherapy, clinical target volume, radiation pneumonia and hematological toxicity had no statistical significance. However, in the arm without CTV, grade 3-4 radiation pneumonia was only $5.5 \%$, compared with $18.0 \%$ in the arm with CTV ( $\mathrm{P}=0.044)$. In conclusion, the smaller target volume for stage III NSCLC treated with IMRT was feasible.

\section{Introduction}

Currently, lung cancer constitutes the major cause of cancer-associated mortality worldwide (1), accounting for $18 \%(2,3)$. Non-small cell lung cancer (NSCLC) accounts for $85 \%$ among lung cancer. The majority of patients are stage III, losing the chance of radical surgery (4). At present, the 1st or 2nd cycle of chemotherapy concurrent with chest irradiation has become the standard treatment for stage III NSCLC in the NCCN Guidelines. However, the lung, regarded as a sensitive organ to radiation damage, is inevitably under irradiation. Radioactive pneumonia is the important restriction factor of radiation dose escalation (5). Numerous studies indicated that radiation pneumonia may be a life-threatening complication $(6,7)$, with a mortality rate of $\sim 4 \%$ (8). Therefore, to reduce the occurrence of radioactive pneumonia is crucial.

At present, clinical target volume (CTV) is a tissue volume that contains gross tumor volume (GTV) and subclinical microscopic malignant lesions following International Commission on Radiation Units and Measurements (ICRU) 62. To take into account $95 \%$ of the microscopic extension, the CTV margin of 8 and $6 \mathrm{~mm}$ must be chosen for adenocarcinoma and squamous cell carcinoma, respectively (9). Delineation of CTV is currently the standard for current intensity-modulated radiotherapy (IMRT) for patients with NSCLC. Cai et al (10) noted that target volume delineation omitting CTV for limited-disease small cell lung cancer receiving IMRT was feasible, and it did not reduce the local control and survival rates, but significantly reduced the incidence of radioactive pneumonia.

Thoracic radiotherapy omitting CTV for stage III NSCLC reduced the radiation volume, so radiation pneumonia may be controlled. The present study compared one arm of patients with CTV and the other arm without CTV with local relapse, distant metastasis, progression-free survival, overall survival and radiation toxicity. The aim of the study was to analyze the feasibility of the smaller target volume for stage III NSCLC treated with IMRT. 


\section{Materials and methods}

Study design. In total, 105 patients with stage III NSCLC who were hospitalized and received IMRT were enrolled. A total of 55 cases were irradiated with target volume without CTV, and 50 cases were irradiated with CTV. All the patients included in the study were followed up at regular intervals: Every 3 months for the first 2 years after treatment, and subsequently every 6 months during 3-5 years. The final follow-up time was November 30, 2012. Follow-up examinations included basic laboratory studies, bone emission computed tomography (ECT), CT of the chest, magnetic resonance imaging (MRI) of the brain and ultrasound imaging of the abdomen. The region that was $5 \mathrm{~mm}$ inside and outside of the planning target volume (PTV) was defined as relapse in-margin. In and out of the area of relapse in-margin were defined as relapse in-field and out-of-field, respectively. Radiation-related toxicity was scored according to the criteria of Radiation Therapy Oncology Group (RTOG).

Patients. In total, 105 patients were treated in the Department of Radiation Oncology at Peking University Cancer Hospital (Beijing, China) between January 1, 2008 to November 30, 2012. All the patients were proved by cytology or histology and were untreated prior to enrollment. Staging procedures included bone ECT, CT of the chest, MRI of the brain and ultrasound imaging of the abdomen. Stage III NSCLC was defined according to the criteria of the 7 th edition of tumor-node-metastasis staging of lung cancer, which was established by the Union for International Cancer Control. Exclusion criteria were receipt of lung cancer resection, recurrence following radiotherapy or metastatic carcinoma.

Treatments. Patients were fixed with a thermoplastic sheet and 5-mm slices were scanned with a conventional CT simulator. The pulmonary extent of the tumor was delineated on pulmonary window (width, $1600 \mathrm{HU}$; level, $-800 \mathrm{HU}$ ) and the mediastinal lymph nodes, which were positive on biopsy or positron emission tomography (PET) or were $\geq 10 \mathrm{~mm}$ in the short axis on the chest CT, and were delineated on mediastinal window (width, $400 \mathrm{HU}$; level, $20 \mathrm{HU}$ ).

In the arm with CTV, target volume was delineated according to ICRU 62. GTV contained a clinically detectable tumor according to CT or PET. The CTV margin of $8 \mathrm{~mm}$ covered microscopic spread of cancer cells in adenocarcinoma, however, this was $6 \mathrm{~mm}$ for squamous cell carcinoma. A margin of 3-15 $\mathrm{mm}$ was added to CTV to form the internal target volume (ITV), which was to cover respiratory movement. Respiratory movement was measured on a conventional simulator during patients' free breathing. Considering setup variations, a margin of $5 \mathrm{~mm}$ was added to ITV to create PTV. In the same way, GTV, ITV and PTV were delineated in the arm without CTV, but CTV was omitted. The treatment planning system used was the Varian Medical System (Palo Alto, CA, USA), and the dose prescription was $100 \%$ PTV for 54-63 Gy/27-35 F/5.4-7 weeks.

Statistical analysis. Statistical analysis was performed with SPSS 17.0 (SPSS, Inc., Chicago, IL, USA). The differences between the two arms were assessed using t-test for the mean
Table I. Patient characteristics.

\begin{tabular}{lccc}
\hline Characteristics & $\begin{array}{c}\text { Arm without } \\
\text { CTV }\end{array}$ & $\begin{array}{c}\text { Arm with } \\
\text { CTV }\end{array}$ & P-value \\
\hline $\begin{array}{l}\text { No. of patients } \\
\text { Gender, n (\%) }\end{array}$ & 55 & 50 & \\
$\quad$ Male & $42(76.4)$ & $40(80.0)$ & 0.653 \\
Female & $13(23.6)$ & $10(20.0)$ & \\
Age, median years & $59(41-78)$ & $61.5(44-81)$ & 0.330 \\
(range) & & & \\
$\leq 65$ years & $38(69.1)$ & $30(60.0)$ & \\
$>65$ years & $17(30.9)$ & $20(40.0)$ & \\
ECOG score, n $(\%)$ & & & \\
0 & $34(61.8)$ & $28(56.0)$ & 0.545 \\
1 & $21(38.2)$ & $22(44.0)$ & \\
Weight loss, n $(\%)$ & & & \\
$>5 \%$ & $3(5.5)$ & $2(4.0)$ & 1.000 \\
$\leq 5 \%$ & $52(94.5)$ & $48(96.0)$ & \\
\hline
\end{tabular}

CTV, clinical target volume; ECOG, Eastern Cooperative Oncology Group.

of sample and using $\lambda^{2}$ test for the constituent ratio of sample. Overall survival and progression-free survival rates were studied by Kaplan-Meier analysis. Patients alive at the time of last follow-up were allocated that date.

\section{Results}

Patients. In the study, 105 cases were evaluated, of which 55 cases were irradiated with target volume without CTV and 50 cases with CTV. All the cases were from the Department of Radiation Oncology at Peking University Cancer Hospital between January 1, 2008 to November 30, 2012. Patient characteristics are listed in Table I.

Chemotherapy cycles. The 1st or 2nd cycle of chemotherapy concurrent with chest irradiation was the standard treatment for stage III NSCLC in the NCCN Guidelines. The arm without CTV accepted $1.54 \pm 1.51$ cycle induction chemotherapy, $0.94 \pm 1.00$ cycle adjuvant chemotherapy and $0.58 \pm 0.91$ cycle concurrent chemotherapy; the arm with CTV accepted $1.31 \pm 1.60$ cycle induction chemotherapy, $0.96 \pm 0.94$ cycle adjuvant chemotherapy and $0.65 \pm 0.95$ cycle concurrent chemotherapy. Treatment delivery was as listed in Table II. There were no statistically significant diffences between the two arms.

GTV and PTV volume and dose, and short-term response. The volume and dose of GTV and PTV, and the short-term response of the two arms are as listed in Table III. There was a statistical significance for PTV volume between the two arms.

Relapse and distant metastases rates. In the arms without and with $\mathrm{CTV}$, the local relapse and distant metastases rates were 32.7 and $32.0 \%(\mathrm{P}=1.000)$, and 56.4 and $48.0 \%(\mathrm{P}=0.946)$, 
Table II. Treatment delivery.

\begin{tabular}{|c|c|c|c|}
\hline Characteristics & Arm without CTV & Arm with CTV & P-value \\
\hline No. of patients & 55 & 50 & \\
\hline \multicolumn{4}{|l|}{ Treatment schedule, n (\%) } \\
\hline Induction chemo & $3(5.5)$ & $6(12.0)$ & \multirow[t]{6}{*}{0.724} \\
\hline Concurrent chemo & $12(21.8)$ & $10(20.0)$ & \\
\hline Induction + concurrent adjuvant chemo & $20(36.4)$ & $19(38.0)$ & \\
\hline Induction + concurrent chemo & $6(10.9)$ & $5(10.0)$ & \\
\hline Concurrent adjuvant & $10(18.2)$ & $5(10.0)$ & \\
\hline RT alone & $4(7.3)$ & $5(10.0)$ & \\
\hline \multicolumn{4}{|l|}{ Induction chemo (cycles), n (\%) } \\
\hline 0 & $26(47.3)$ & $20(40.0)$ & \multirow[t]{4}{*}{0.828} \\
\hline 1 & $4(7.3)$ & $3(6.0)$ & \\
\hline 2 & $16(29.1)$ & $16(32.0)$ & \\
\hline$\geq 3$ & $9(16.4)$ & $11(22.0)$ & \\
\hline \multicolumn{4}{|l|}{ Concurrent chemo (cycles), n (\%) } \\
\hline 0 & $37(67.3)$ & $35(70.0)$ & \multirow[t]{3}{*}{0.517} \\
\hline 1 & $0(0)$ & $1(2.0)$ & \\
\hline 2 & $18(32.7)$ & $14(28.0)$ & \\
\hline \multicolumn{4}{|l|}{ Adjuvant chemo (cycles), n (\%) } \\
\hline 0 & $25(45.5)$ & $26(52.0)$ & \multirow[t]{3}{*}{0.117} \\
\hline 1 & $7(12.7)$ & $1(2.0)$ & \\
\hline 2 & $23(41.8)$ & $23(46.0)$ & \\
\hline
\end{tabular}

CTV, clinical target volume; chemo, chemotherapy; RT, radiation therapy.

respectively. Relapse and metastasis sites of the two arms are listed in Table IV.

Toxicities. Radiation and chemo-related toxicities were scored according to RTOG. They are listed in Table V. The only apparent statistical significance between the two arms was for grade $0-2$ radiation pneumonia.

\section{Discussion}

Once radiation pneumonia occurs during radiotherapy, the radiotherapy must be terminated. Thus, it increases the local relapse and reduces the overall survival rates, influences the patient's quality of life and can even result in fatality. The 1st or 2nd cycle of chemotherapy concurrent with chest irradiation has become the standard treatment for stage III NSCLC in the NCCN Guidelines. It also increases the incidence of radiation pneumonia at the same time as improving the curative effect (11). In the study by Sura et al (12), IMRT increased the volume of lung receiving smaller, yet potentially toxic doses of radiation, thus it increased the incidence of radiation pneumonia. Therefore, more attention must be paid to reduce the incidence of radiation pneumonia.

How to reduce the incidence of radiation pneumonia whilst not reducing the radiotherapy curative effect remains to be solved. Cai et al (10) noted that target volume delineation omitting CTV for limited-disease small cell lung cancer receiving IMRT was feasible, and it did not increase the local
Table III. Volume and dose of GTV and PTV, and short-term response of the two arms.

\begin{tabular}{lccc}
\hline Characteristics & $\begin{array}{c}\text { Arm without } \\
\text { CTV }\end{array}$ & $\begin{array}{c}\text { Arm with } \\
\text { CTV }\end{array}$ & P-value \\
\hline Volume, $\mathrm{cm}^{3}$ & & & \\
GTV & $168.48 \pm 70.40$ & $166.84 \pm 75.76$ & 0.909 \\
PTV & $370.71 \pm 117.94$ & $471.59 \pm 154.01$ & 0.000 \\
Dose, Gy & & & \\
GTV & $61.56 \pm 2.34$ & $61.84 \pm 3.12$ & 0.607 \\
PTV & $58.91 \pm 3.46$ & $58.48 \pm 3.56$ & 0.533 \\
Short-term & & & \\
response, $\mathrm{n}$ & & & \\
CR & 4 & 3 & 0.961 \\
PR & 32 & 29 & \\
SD & 19 & 18 & \\
PD & 0 & 0 & \\
\end{tabular}

CTV, clinical target volume; GTV, gross tumor volume; PTV, planned target volume; CR, complete response; PR, partial response; SD, stable disease; PD, progressive disease.

relapse rate or reduce the survival rate, however, it significantly reduced the incidence of radiation pneumonia. Comparing the arm without CTV to the arm with CTV, the local relapse rate 
Table IV. Relapse and metastasis sites of the two arms.

\begin{tabular}{lccc}
\hline Characteristics & $\begin{array}{c}\text { Arm without } \\
\text { CTV }\end{array}$ & $\begin{array}{c}\text { Arm with } \\
\text { CTV }\end{array}$ & P-value \\
\hline No. of patients & 55 & 50 & \\
Local relapse, n (\%) & $18(32.7)$ & $16(32.0)$ & 1.000 \\
In field & $17(30.9)$ & $15(30.0)$ & \\
In margin & $1(1.8)$ & $1(2.0)$ & \\
Metastasis, n (\%) & $31(56.4)$ & $24(48.0)$ & 0.946 \\
Brain & $6(10.9)$ & $4(8.0)$ & \\
Bone & $7(12.7)$ & $5(10.0)$ & \\
Liver & $7(12.7)$ & $4(8.0)$ & \\
Lung & $3(5.5)$ & $2(4.0)$ & \\
Mediastina LN & $2(3.6)$ & $3(6.0)$ & \\
Peritoneum LN & $0(0.0)$ & $1(2.0)$ & \\
Supraclavicular LN & $1(1.8)$ & $1(2.0)$ & \\
Adrenal gland & $1(1.8)$ & $1(2.0)$ & \\
\hline
\end{tabular}

CTV, clinical target volume; LN, lymph node.

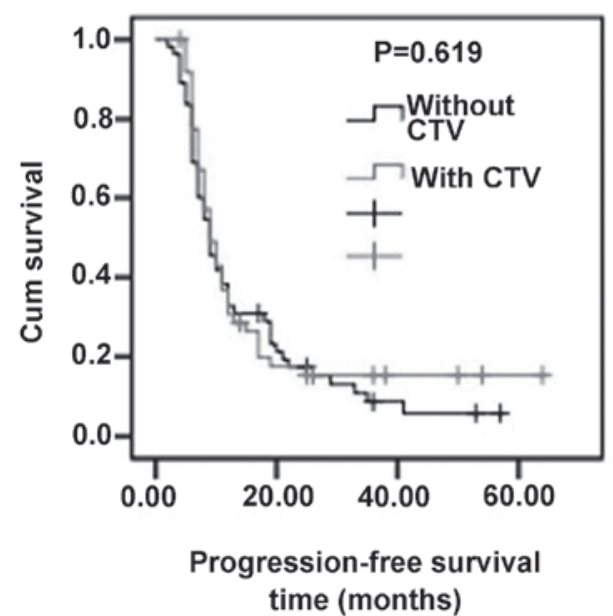

Figure 1. Kaplan-Meier analysis of progression-free survival. The median progression-free survival of the two arms were 9 months $(\mathrm{P}=0.619)$. The difference of progression-free survival of the two arms had no statistical significance.

was 16.7 vs. $17.1 \%(\mathrm{P}=0.586)$, and distant metastases rate was 42.6 vs. $51.4 \%(\mathrm{P}=0.274)$. The 1 -, 2 - and 3 -year survival rates of the arm without CTV and arm with CTV were 81.0, 66.2 and $61.5 \%$, and $88.6,61.7$ and $56.6 \%(\mathrm{P}=0.517)$, respectively. In the arm without CTV and arm with CTV, grade 3-4 radiation pneumonia was 7.4 and $22.9 \%(\mathrm{P}=0.040)$, respectively. Whether this can be applied to NSCLC is unknown.

To the best of our knowledge, this is the first clinical study reporting target volume omitting CTV in stage III NSCLC treated with IMRT. We were concerned most with whether omitting CTV resulted in a high margin local relapse rate. In the study, the margin local relapse rate was $1.8 \%$ in the arm without CTV and $2.0 \%$ in the arm with CTV. In the two arms, the major local relapse section was all in-field. The existence of hypoxic cancer cells, which are radiation resistant and require higher irradiation doses to be killed (13), was the possible
Table V. Radiation and chemo-related toxicities of the two arms.

\begin{tabular}{lcrr}
\hline & $\begin{array}{c}\text { Arm without } \\
\text { Toxicities }\end{array}$ & $\begin{array}{c}\text { Arm with } \\
\text { CTV, n (\%) }\end{array}$ & P-value \\
\hline Hematological toxicity & & & \\
$0-2$ & $49(89.1)$ & $42(84.0)$ & 0.443 \\
$3-4$ & $6(10.9)$ & $8(16.0)$ & \\
Radiation esophagitis & & & \\
0-2 & $50(90.9)$ & $43(86.0)$ & 0.430 \\
$3-4$ & $5(9.1)$ & $7(14.0)$ & \\
Radiation pneumonia & & & \\
$0-2$ & $52(94.5)$ & $41(82.0)$ & 0.044 \\
$3-4$ & $3(5.5)$ & $9(18.0)$ & \\
\hline CTV, clinical target volume. & & & \\
\hline
\end{tabular}

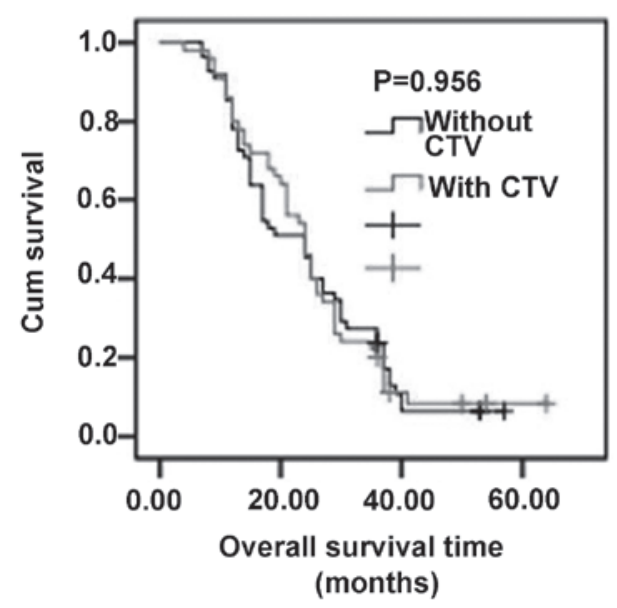

Figure 2. Kaplan-Meier analysis of overall survival. The 1-, 2- and 3-year survival rates of the arm without clinical target volume (CTV) and arm with CTV were 74.5, 43.6 and $23.6 \%$, and 70.0, 46.0 and $20.0 \%(\mathrm{P}=0.956)$, respectively. The difference of overall survival of the two arms also had no statistical significance.

reason. However, in the arm without CTV, grade 3-4 radiation pneumonia was only $5.5 \%$, compared with $18.0 \%$ in the arm with CTV $(\mathrm{P}=0.044)$.

The possible reasons for these conclusions were as follows. First, the radiation dose is one of the important prognostic factors. Omitting CTV can reduce the radiation target volume and make it easier for GTV to receive an efficient radiation dose, which can reduce the local relapse (14-16). GTV could receive a higher radiation dose, which can eliminate the hypoxic cancer cells, whilst simultaneously protecting the normal tissue. Therefore, a smaller target volume and higher GTV radiation dose may be the future research direction. Second, the subclinical lesions that were scattered on the edge of the GTV had a lower tumor burden and were in an oxygen enrichment condition. Therefore, their radiation sensitivity was better (17). In radiotherapy, the incidental dose to the ipsilateral hilus pulmonis, the mediastinal and paratracheal nodes could be $<40-50$ Gy when these regions were not in the radiotherapy target volume (18). The subclinical lesions received enough to 
be eliminated by the incidental dose. As $100 \%$ PTV irradiation was the prescription dose in the present study, $\mathrm{a} \geq 8 \mathrm{~mm}$ range between PTV and GTV would accept at least half of the total radiation dose. Third, NSCLC was not radiation sensitive, but had easy local relapse and distant metastases compared with SCLC (19). The main cause of treatment failure was local recurrence and distant metastasis, however, the main cause of fatality was always organ failure lead by distant metastasis (20). In the present study, the distant metastasis rate of the two arms was significantly higher compared with the local recurrence rate. Therefore, a perfect local control rate is insignificant if the higher distant metastasis rate cannot be reduced.

In conclusion, the smaller target volume for stage III NSCLC treated with IMRT was feasible. It did not increase the local relapse rate and reduce survival rate, but significantly reduced the incidence of radiation pneumonia. However, the study is not a randomized controlled study, and further research and validation is necessary.

\section{References}

1. Siegel R, Naishadham D and Jemal A: Cancer statistics, 2013. J Clin 63: 11-30, 2013.

2. Jemal A, Siegel R, Xu J and Ward E: Cancer statistics, 2010. CA Cancer J Clin 60: 277-300, 2010.

3. Jemal A, Bray F, Center MM, Ferlay J, Ward E and Forman D: Global cancer statistics. CA Cancer J Clin 61: 69-90, 2011.

4. Sant M, Aareleid T, Berrino F, Bielska Lasota M, Carli PM, Faivre J, Grosclaude P, Hédelin G, Matsuda T, Møller H, et al; EUROCARE Working Group: EUROCARE-3: Survival of cancer patients diagnosed 1990-94 - results and commentary. Ann Oncol 14 (Suppl 5): v61-v118, 2003.

5. Semenenko VA, Molthen RC, Li C, Morrow NV, Li R, Ghosh SN, Medhora MM and Li XA: Irradiation of varying volumes of rat lung to same mean lung dose: A little to a lot or a lot to a little? Int J Radiat Oncol Biol Phys 71: 838-847, 2008.

6. Yorke ED, Jackson A, Rosenzweig KE, Merrick SA, Gabrys D, Venkatraman ES, Burman CM, Leibel SA and Ling CC: Dose-volume factors contributing to the incidence of radiation pneumonitis in non-small-cell lung cancer patients treated with three-dimensional conformal radiation therapy. Int J Radiat Oncol Biol Phys 54: 329-339, 2002.

7. Kong FM, Hayman JA, Griffith KA, Kalemkerian GP, Arenberg D, Lyons S, Turrisi A, Lichter A, Fraass B, Eisbruch A, et al: Final toxicity results of a radiation-dose escalation study in patients with non-small-cell lung cancer (NSCLC): Predictors for radiation pneumonitis and fibrosis. Int J Radiat Oncol Biol Phys 65: 1075-1086, 2006.

8. Marks LB, Bentzen SM, Deasy JO, Kong FM, Bradley JD, Vogelius IS, El Naqa I, Hubbs JL, Lebesque JV, Timmerman RD, et al: Radiation dose-volume effects in the lung. Int J Radiat Oncol Biol Phys 76 (Suppl): S70-S76, 2010.
9. Giraud P, Antoine M, Larrouy A, Milleron B, Callard P, De Rycke Y,Carette MF, Rosenwald JC, Cosset JM, Housset M, et al: Evaluation of microscopic tumor extension in non-small-cell lung cancer for three-dimensional conformal radiotherapy planning. Int J Radiat Oncol Biol Phys 48: 1015-1024, 2000

10. Cai S, Shi A, Yu R and Zhu G: Feasibility of omitting clinical target volume for limited-disease small cell lung cancer treated with chemotherapy and intensity-modulated radiotherapy. Radiat Oncol 9: 17, 2014.

11. Parashar B, Edwards A, Mehta R, Pasmantier M, Wernicke AG, Sabbas A, Kerestez RS, Nori D and Chao KS: Chemotherapy significantly increases the risk of radiation pneumonitis in radiation therapy of advanced lung cancer. Am J Clin Oncol 34: 160-164, 2011.

12. Sura S, Gupta V, Yorke E, Jackson A, Amols H and Rosenzweig KE: Intensity-modulated radiation therapy (IMRT) for inoperable non-small cell lung cancer: The Memorial Sloan-Kettering Cancer Center (MSKCC) experience. Radiother Oncol 87: 17-23, 2008

13. Karar J and Maity A: Modulating the tumor microenvironment to increase radiation responsiveness. Cancer Biol Ther 8: 1994-2001, 2009.

14. Rengan R, Rosenzweig KE, Venkatraman E, Koutcher LA, Fox JL, Nayak R, Amols H, Yorke E, Jackson A, Ling CC, et al: Improved local control with higher doses of radiation in large-volume stage III non-small-cell lung cancer. Int J Radiat Oncol Biol Phys 60: 741-747, 2004.

15. Bradley JD, Ieumwananonthachai N, Purdy JA, Wasserman TH, Lockett MA, Graham MV and Perez CA: Gross tumor volume, critical prognostic factor in patients treated with three-dimensional conformal radiation therapy for non-small-cell lung carcinoma. Int J Radiat Oncol Biol Phys 52: 49-57, 2002.

16. Werner-Wasik M, Scott C, Cox JD, Sause WT, Byhardt RW, Asbell S, Russell A, Komaki R and Lee JS: Recursive partitioning analysis of 1999 Radiation Therapy Oncology Group (RTOG) patients with locally-advanced non-small-cell lung cancer (LA-NSCLC): Identification of five groups with different survival. Int J Radiat Oncol Biol Phys 48: 1475-1482, 2000.

17. Littbrand B and Révész L: The effect of oxygen on cellular survival and recovery after radiation. Br J Radiol 42: 914-924, 1969.

18. Zhao L, Chen M, Ten Haken R, Chetty I, Chapet O, Hayman JA and Kong FM: Three-dimensional conformal radiation may deliver considerable dose of incidental nodal irradiation in patients with early stage node-negative non-small cell lung cancer when the tumor is large and centrally located. Radiother Oncol 82: 153-159, 2007.

19. Jemal A, Siegel R, Ward E, Hao Y, Xu J, Murray T and Thun MJ: Cancer statistics, 2008. CA Cancer J Clin 58: 71-96, 2008.

20. Arriagada R, Le Chevalier T, Quoix E, Ruffie P, de Cremoux H, Douillard JY, Tarayre M, Pignon JP and Laplanche A: ASTRO (American Society for Therapeutic Radiology and Oncology) plenary: Effect of chemotherapy on locally advanced non-small cell lung carcinoma: a randomized study of 353 patients. GETCB (Groupe d'Etude et Traitement des Cancers Bronchiques), FNCLCC (Féderation Nationale des Centres de Lutte contre le Cancer) and the CEBI trialists. Int J Radiat Oncol Biol Phys 20: 1183-1190, 1991. 